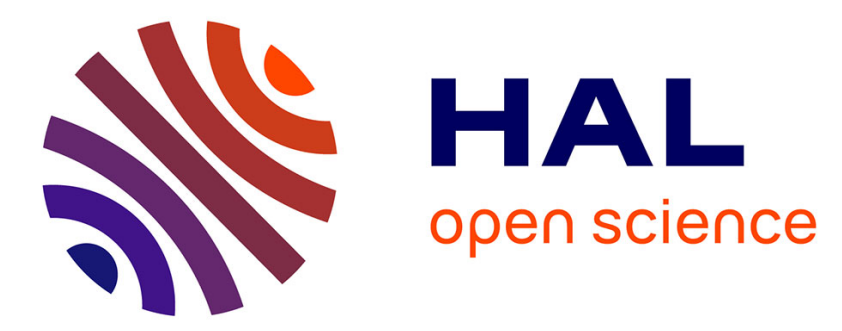

\title{
Pour une définition générale de l'intensité dans le langage
}

Clara Romero

\section{To cite this version:}

Clara Romero. Pour une définition générale de l'intensité dans le langage. Travaux de Linguistique: Revue Internationale de Linguistique Française, 2007, Travaux de linguistique, 2007-1 (54), pp.57-68. halshs-00166172

\section{HAL Id: halshs-00166172 https://shs.hal.science/halshs-00166172}

Submitted on 19 Oct 2011

HAL is a multi-disciplinary open access archive for the deposit and dissemination of scientific research documents, whether they are published or not. The documents may come from teaching and research institutions in France or abroad, or from public or private research centers.
L'archive ouverte pluridisciplinaire HAL, est destinée au dépôt et à la diffusion de documents scientifiques de niveau recherche, publiés ou non, émanant des établissements d'enseignement et de recherche français ou étrangers, des laboratoires publics ou privés. 
Titre : Pour une définition générale de l'intensité dans le langage

Auteur : Clara Romero / clara.romero@ parisdescartes.fr

Référence : (2007) Travaux de linguistique 54, p. 57-68

Résumé :

L'intensité est un domaine qui a souvent été étudié dans les différentes langues, notamment par les grammairiens, sans pourtant qu'un consensus ne se dégage quant à la définition de ce terme. Nous tentons ici, à travers un panorama de tous les faits linguistiques que peut recouvrir cette notion dans le langage (sous différentes étiquettes, telles que haut degré, mise en relief, etc.) de souligner ce qui en fait finalement l'unité, et qui la définit : l'idée de tension, d'écart que l'on appréhende. Celui-ci peut être d'ordre quantitatif, ce qui renvoie à la scalarité et au degré, ou qualitatif, il peut alors être actuel ou virtuel.

Abstract :

Intensity is a field that has often been studied in various languages, notably by grammarians, however without a consensus being ever reached as to the definition of this term. By taking a broad view of all the linguistic facts that may be encompassed (under different labels, such as high degree, emphasis, etc.) by the notion of intensity in language, we attempt to highlight what ultimately unites and defines it: The concept of tension, and/or of gap that may be taken in. This gap can be classified as quantitative, relating to degree and scalarity; or as qualitative, being in such case real or virtual.

NB : Le présent document est un manuscrit. Pour des raisons techniques, des caractères spéciaux ont pu subir des dommages. En cas de besoin, veuillez vous reporter à la version publiée.

\section{Introduction}

Le degré d'intensité est une notion fort ancienne, sur laquelle on n'a pas manqué de se pencher depuis que l'on a entrepris de décrire les langues. À l'heure actuelle, toute grammaire comporte un chapitre, plus ou moins étendu selon la taille de l'ouvrage, consacré à l'expression du degré d'intensité. Chevalier \& alii (1994: 198) remarquent à juste titre : "L'intensité forte est celle qui dispose du plus grand nombre de moyens d'expression stylistiques" et ajoutent exclamativement, avant de citer les plus notables d'entre eux, que "certains observateurs vont jusqu'à distinguer une vingtaine de rubriques !". Mais quel que soit le nombre de rubriques que l'on distinguera, les quelques moyens qu'une grammaire a vocation à présenter comme réservés à l'expression de l'intensité seront bien peu de chose comparés à la grande richesse de procédés réellement mis à disposition par la langue et exploités par les locuteurs.

À propos de la diversité de ces procédés, le linguiste Reinhard Kiesler écrivait (2000), pour inaugurer un article intitulé "Où en sont les études sur la mise en relief ?", sur lequel je m'appuierai tout au long de cette introduction :

La mise en relief est un des phénomènes les plus quotidiens dans l'usage des langues naturelles, et le concept de "mise en relief" est une des notions fondamentales de la linguistique. Néanmoins, elle n'a jamais été définie de façon explicite. La plupart des dictionnaires de linguistique n'ont pas d'entrée correspondante [...]. Souvent, même les ouvrages spécialisés n'en offrent pas de définition ou, alors, en donnent de vagues ou contradictoires. 
Au fond, l'auteur soulève deux problèmes majeurs qui se rejoignent : le problème de la définition et celui de la terminologie. J'utilise pour ma part le mot intensité pour parler globalement de divers faits linguistiques que l'on a pu appeler (je cite toujours Kiesler) : mise en relief / évidence / lumière / valeur / vedette, emphase, intensification, renforcement, topicalisation, focalisation, accentuation, insistance, saillance, mais -et c'est là que le bât blesse- avec des définitions différentes selon les auteurs ! Kiesler, lui, utilise le terme général de mise en relief.

J'ai, pour ma part, dans une thèse de doctorat (Romero, 2001), "passé au peigne fin" et en recherchant l'exhaustivité, les différents moyens de s'exprimer intensément en français. Cela m'a permis d'en faire ressortir les caractères communs -tant au plan du contenu qu'à celui de l'expression-. J'espère donc modestement désormais être en mesure de proposer une définition globale des faits relevant de l'intensité dans le langage, que Kiesler appelle de ses vœux. Mais d'abord, il me faut prendre acte des définitons que donne Kiesler lui-même :

Un énoncé neutre, non marqué (En) est modifié (transformé), par un processus de modification au moyen de procédés de mise en relief (x), en un énoncé mettant en relief, marqué (Em) ; la 'mise en relief' $(\mathrm{H})$ consiste en ce processus.

Cette première définition porte, me semble-t-il, sur l'expression. Je l'ai volontiers adoptée dans ma thèse, en retenant l'idée que l'intensification $(H)$ est une fonction, donc : $H(E n)=E m$. À noter cependant qu'au niveau du contenu, j'opposerai plus volontiers un contenu non intense, à un contenu intense.

La deuxième définition que propose Kiesler, est d'ordre pragmatique, voire psychologique. Je la cite partiellement :

La mise en relief est toute transformation s'effectuant pendant un processus de production linguistique, laquelle se manifeste sur le plan locutionnaire de l'énoncé - qui, orienté téléologiquement de façon consciente ou inconsciente, a pour but une intensification de l'effet perlocutionnaire visé par l'énoncé.

Là encore, je suis en accord avec une telle définition, même s'il ne faut pas omettre que ceci se fait via une étape à mon avis proprement linguistique, c'est-à-dire celle de l'acte illocutionnaire. Mais mon approche se situera ici au niveau du contenu, c'est-à-dire en opposant un contenu non intense à un contenu intense.

\section{L'intensité comme écart entre deux états, appréhendé comme tel}

Au plan le plus abstrait, l'intensité est d'abord une tension (v. le même radical tenssur lequel ces mots sont formés). Autrement dit, l'intensité d'un phénomène $\mathbf{X}$ résulte de l'appréhension ${ }^{1}$ de l'écart (ou de la différence) entre deux états $x_{1}$ et $x_{2}$ relatifs à ce phénomène. De fait, cette définition n'entre pas en contradiction avec le sens que ce mot a ordinairement.

Tout écart se caractérise par une certaine amplitude dans le contraste. Tout écart comporte donc conjointement ces deux aspects : amplitude, contraste. Cependant, celui-ci peut être cognitivement appréhendé sous l'un de ces aspects plutôt que l'autre, selon ce qui retient l'attention. On peut appeler quantitatif l'aspect qui correspond à l'amplitude, et qualitatif celui qui correspond au contraste. Retenons donc d'ores et déjà (ceci sera développé

\footnotetext{
${ }^{1}$ Ou l'ex-pression de l'écart, si l'on veut.
} 
dans les $\S 2$ et 3 ) que les différentes modalités ou phénomènes d'intensité s'articulent autour de ces deux pôles, et que leur point commun est la notion de tension, fondée sur celle d'écart.

Cependant, non seulement les deux aspects sont toujours sous-jacents, mais de plus, les notions de quantité et de qualité, bien que conceptuellement séparées, interfèrent en pratique. Ainsi, l'écart entre un citron et une orange est vu comme qualitatif (différence de nature), mais il est tout de même quantifiable ; il est plus petit que celui qui existe entre un citron et un chat. De même, l'écart entre des températures de $-5^{\circ} \mathrm{C}$ et de $30^{\circ} \mathrm{C}$ est d'abord considéré comme quantitatif (différence de degré), à ceci près qu'il entraine ce qu'on appelle un saut qualitatif: à $-5^{\circ} \mathrm{C}$ l'eau est (à pression atmosphérique) solide, et à $30^{\circ} \mathrm{C}$ elle est liquide. Cette interdépendance conceptuelle a des conséquences sur le plan linguistique ${ }^{2}$ (v. $\S$ 4). Remarquons pour l'instant simplement que si le mot trempé est intense, car il présente un écart qu'on se figure comme quantitatif par rapport à mouillé, on a le choix entre : C'est mouillé, c'est même trempé pour mettre l'accent sur cet aspect quantitatif de l'écart, et : C'est pas mouillé, c'est trempé, pour insister sur l'aspect qualitatif, en illustrant l'idée de saut qualitatif.

\section{L'écart quantitatif (ou l'intensité d'ordre quantitatif)}

La quantification implique la notion d'échelle. Une échelle est ce qui permet de mesurer une quantité. On peut la définir comme suit : une échelle est un ensemble a priori infini d'éléments ordonnés ${ }^{3}$ dont un plus petit, schématisable ainsi :

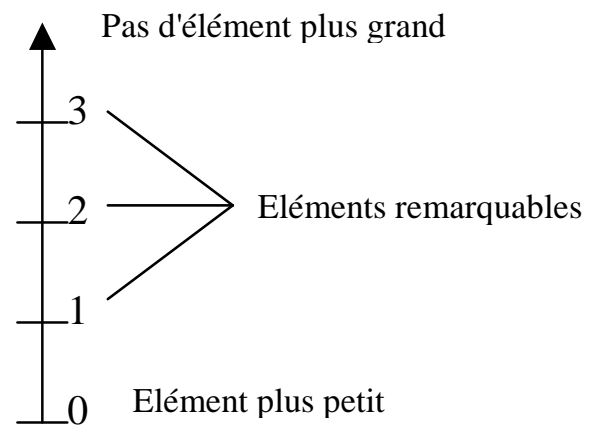

On appellera relation d'ordre (entre deux propositions A et B), la relation suivante :

$(\mathrm{B}>\mathrm{A}) \Leftrightarrow((\mathrm{B} \Rightarrow \mathrm{A}) \wedge \neg(\mathrm{A} \Rightarrow \mathrm{B}))$

Il ne s'agit pas de la définition mathématique de la relation d'ordre ${ }^{4}$, mais elle s'y apparente, et elle est suffisante pour étudier des énoncés. Je l'emprunte, en l'adaptant, à Moescheler et

\footnotetext{
${ }^{2}$ C'est ce qui explique la confusion terminologique, le fait que, par exemple, on appelle mise en relief des faits linguistiques qui semblent bien différents.

${ }^{3}$ S'agissant de faits de langage, il me semble qu'il est préférable de concevoir l'ordre comme étant partiel. Total signifierait que l'on peut ordonner tous les éléments. Or, certains énoncés intenses se distinguent par autre chose que le degré (opposition très / si). D'autre part, il doit être vu comme dense. Cela signifie qu'il existe toujours un élément entre deux éléments quelconques. L'ensemble a alors la puissance du continu, c'est-à-dire que l'on peut établir une bijection entre cet ensemble et l'ensemble $\rho$. En termes plus courants, il y a sur l'échelle une infinité de positions possibles, ce qui offre plus souplesse, par exemple pour l'analyse de de plus en plus.

${ }^{4}$ En termes mathématiques : Une relation est une propriété qui porte sur l'ensemble des couples formés d'un élément d'un premier ensemble et d'un élément d'un second. Soit $\Re$ une relation binaire sur un ensemble $\mathrm{E} ; \mathfrak{R}$
} 
Reboul (1994 : 197) $)^{5}$, qui définissent ainsi une échelle quantitative. Ainsi par exemple, si l'on doit ordonner les propositions :

A : Je vais à la fac lorsque j'ai cours

$\mathrm{B}$ : Je vais à la fac tous les jours

On trouve que $\mathrm{B}>\mathrm{A}$ car

Je vais à la fac tous les jours $\Rightarrow$ Je vais à la fac lorsque j'ai cours

$\wedge \neg$ Je vais à la fac lorsque j'ai cours $\Rightarrow$ Je vais à la fac tous les jours

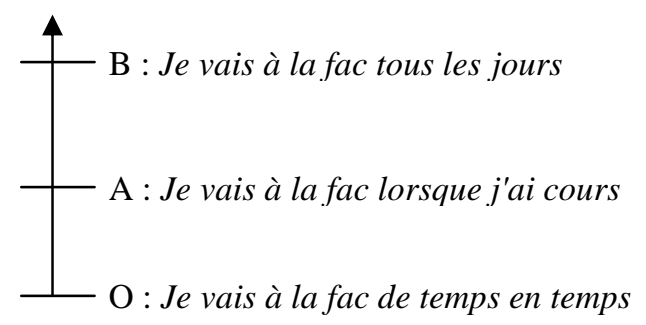

Cela revient pour nous à dire que $\mathrm{B}$ est plus intense que $\mathrm{A}$, lui-même plus intense que O. Mais s'agissant de faits linguistiques, c'est-à-dire de discours, véhiculant du sens, et non simplement de quantités objectivées (lesquelles ne revêtent en elles-mêmes aucune intensité), il faut souligner que l'intensité n'émerge qu'en considérant l'écart plus ou moins grand entre ce qui est (élément actualisé, en particulier s'il est grand) et ce qui pourrait être (éléments impliqués par lui, en particulier le plus petit). Autrement dit $\mathrm{O}$, A et $\mathrm{B}$ ne sont ordonnées que parce que, conformément à une conception dite argumentativiste de la langue (v. Ducrot, 1984), elles pointent vers un sens (elles tendent vers une conclusion), en l'occurrence, quelque chose comme : Je suis un étudiant assidu, et c'est compte tenu de ce sens que B est plus intense que $\mathrm{O}$. Ainsi les propositions $\mathrm{A}$ et $\mathrm{B}$ et $\mathrm{O}$ peuvent-elles être absolument quelconques, ne faisant pas appel à de la quantité ${ }^{6}$, ou même inversant l'ordre absolu des quantités, tant qu'on peut les situer sur la même échelle discursive.

Précisons à présent la distinction traditionnellement faite entre quantité, au sens courant et restreint du terme, et degré. La notion générale de quantité peut s'appliquer à la substance (quantité de substance : sens courant de "quantité") ou à la qualité (quantité de qualité : on parle alors de degré, ou d'intensité ${ }^{7}$ ).

L'intensité d'ordre quantitatif concerne tout ce qui relève de la gradation, de la scalarité, du degré qu'on a pu appeler "absolu", par opposition aux degrés de comparaison. D'autres distinctions concernant le mode de quantification sont classiques : massif / comptable, et quantification interne (= requantification de quelque chose qui a été préalablement quantifié) / quantification externe. Pour résumer, voici quelques formes linguistiques de base correspondantes :

est une relation d'ordre si et seulement si : $\Re$ est réflexive (= pour tout élément a de $\mathrm{E}$, on a a $\Re$ a), antisymétrique (= pour tout couple $(\mathrm{a}, \mathrm{b})$ d'éléments de $\mathrm{E}$, si $\mathrm{a} \Re \mathrm{b}$ et $\mathrm{b} \Re \mathrm{a}$, alors $\mathrm{a}=\mathrm{b}$ ), transitive (= pour tout triplet $(\mathrm{a}, \mathrm{b}, \mathrm{c}$ )

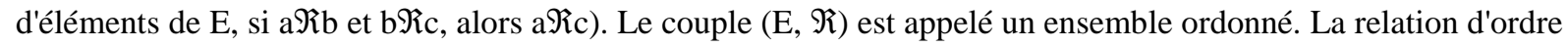
habituelle dans $\rho$ est notée $\leq$. (D'après Delelicq, $1998: 543,558$ )

${ }^{5}$ Pour ces auteurs, une échelle quantitative est un ensemble ordonné de prédicats $\left\langle\mathrm{e}_{1}, \mathrm{e}_{2}, \mathrm{e}_{3}, \ldots \mathrm{e}_{\mathrm{n}}\right\rangle$ tel que, si $\mathrm{A}$ est un cadre syntaxique et $A\left(e_{i}\right)$ une phrase bien formée, $A\left(e_{1}\right)$ implique $A\left(e_{2}\right), A\left(e_{2}\right)$ implique $A\left(e_{3}\right)$, mais pas l'inverse.

${ }^{6}$ L'exemple maintes fois repris de Ducrot était (cité de mémoire) : A : Jacques et venu, B : (Même) Pierre est venu, Conclusion: C'était une fête réussie.

${ }^{7} \mathrm{Ce}$ mot a alors un sens plus restreint que celui que j'emploie, qui permet à certains d'opposer intensité (quantité de qualité) et extensité (quantité de substance). 


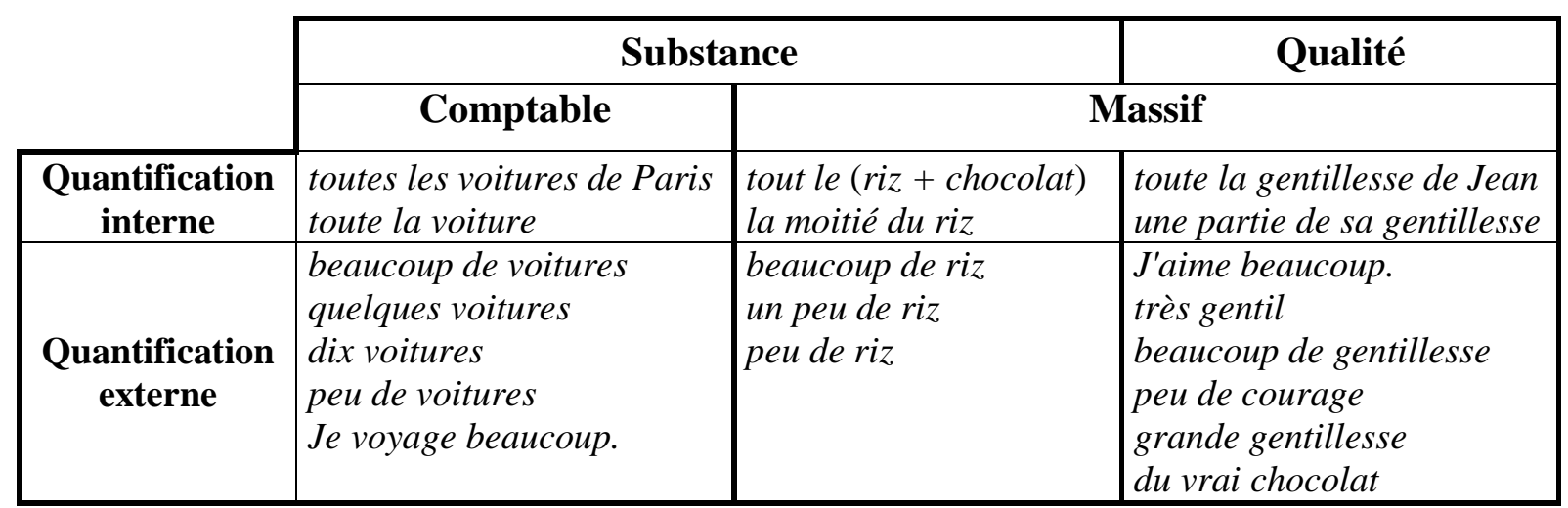

Ce tableau appelle quelques remarques : tout d'abord on notera que la qualité n'est pas réservée aux adjectifs (gentil) ou aux noms dérivés d'adjectifs (gentillesse), mais s'étend aux autres noms (chocolat), également concçus comme des prédicats scalaires.

Ensuite, constatation qui illustre les limites de cette dichotomie parmi ce à quoi peut s'appliquer la quantité, ${ }^{8}$ on aurait du mal à classer dans ce tableau l'intensification par beaucoup de certains verbes. Dans boire beaucoup ou gagner beaucoup on peut considérer que beaucoup s'applique au complément d'objet implicite du verbe (par ex. "beaucoup d'eau", ou "beaucoup d'argent") ; il s'agit donc de quantité de substance. Sortir beaucoup, voyager beaucoup, danser beaucoup, avoir beaucoup vécu à Paris, concernent plus nettement l'aspect duratif ou fréquentatif (itératif) de l'action, ou les deux à la fois. La quantité s'applique donc à nouveau à une substance, qui est le temps (d'ailleurs, est-il alors massif ou comptable ?). Mais un cas comme souffrir beaucoup, montre que beaucoup est susceptible de concerner aussi bien l'aspect duratif ou fréquentatif que l'intensité de la souffrance, ou même les trois à la fois. Ainsi, en se référant toujours à une conception argumentativiste de la langue, on peut douter de la pertinence de la distinction substance / qualité pour une définition de l'intensité. En effet, beaucoup fait dans tous les cas tendre plus l'énoncé vers la conclusion.

Insistions enfin sur le fait que la quantification est une opération fondamentale dans le langage qui n'entraine pas forcément de l'intensité. La comparaison véritable (non intensive) par exemple, pas plus que la détermination du nom, ne relèvent de l'intensité, même si ces opérations mettent en jeu la quantification. Un autre point essentiel à cet égard, est donc que l'énoncé est intense :

- seulement si l'écart qui le constitue est vu comme tel : on pose un écart par rapport à un état considéré comme non intense (d'ailleurs relatif et subjectif)

- d'autant plus que cet écart est vu comme grand. Plus l'écart est petit, moins il existe, et moins la notion d'intensité est pertinente (opératoire) pour en parler. Ainsi,

1) Des énoncés tels que Il y avait dix voitures, ou J'ai acheté $10 \mathrm{kgs}$ de fromage n'ont en soi rien d'intense.

2) Pareillement, même si l'on a raison de rapporter les morphèmes quelques, un peu (par exemple) à la scalarité, parce qu'ils s'opposent à beaucoup, ils ne sont pas des intensifs, parce qu'ils ne posent pas un écart. Beaucoup en revanche, et même peu posent un écart par rapport

\footnotetext{
${ }^{8}$ Celle-ci m'a été inspirée par la communication de Silvia Aldler \& Maria Asnes.
} 
à un état neutre, plus attendu, une norme. ${ }^{9}$ Dans le même ordre d'idées, l'énoncé Je ne suis pas fatigué, est une quantification a priori neutre, dénuée d'intensité, et qui, au surplus, peut passer pour un état normal. Dans certaines circonstances, après une activité fatigante, il est possible que l'état de non fatigue constitue un écart avec ce qui est attendu, on aura alors une intonation particulière, on dira Je ne suis pas du tout fatigué, énoncé intense, ou autre chose propre à marquer cet écart.

\section{L'écart qualitatif (ou l'intensité d'ordre contrastif)}

La qualification est elle aussi une opération fondamentale non nécessairement intense. Par qualification, j'entends plus largement dénomination, ou encore le fait d'attribuer un signe à un référent. Là encore, l'expression n'est intense que lorsque l'écart aboutit à une opposition explicite. ${ }^{10}$ Il est important de distinguer deux types de contraste selon que l'opposition a lieu sur l'axe syntagmatique (il sera dit actuel) ou paradigmatique (il sera dit virtuel).

Le contraste actuel est présent, par exemple, dans les figures d'opposition telles que l'antithèse, le paradoxisme, l'oxymore, etc. où les éléments qui s'opposent (diamétralement) apparaissent en même temps. Cela ressortit, donc, à la fonction poétique du langage, selon Jakobson $(1963: 220)$ :

La fonction poétique projette le principe d'équivalence de l'axe de la sélection sur l'axe de la combinaison.

À ceci près, que c'est plutôt du côté du signifié que du signifiant qu'il faut rechercher lesdites équivalences concernant l'intensité résidant dans le contraste actuel. Quelques exemples de ces figures, où l'intensité ressort du contraste :

Ton bras est invaincu, mais non pas invincible <Le Cid>
Quoi ce royal croquand, ce maraud couronné ? <V. Hugo>
Plus ça change, plus c'est la même chose. <A. Karr $>$
Etre cuisinier et ne pas savoir monter une mayonnaise! <Culioli >11

$\grave{A}$ un autre niveau de la description du langage (communicationnel, pragmatique), on peut même envisager que l'écart se trouve entre les interlocuteurs en présence. Au MoyenÂge, un genre poétique dialogué appelé tenson opposait des interlocuteurs sur un sujet donné. Nul doute que, comme semble l'indiquer ce terme, leurs échanges regorgeaient d'intensité. D'ailleurs, l'étymologie du verbe tancer ramène elle aussi à l'idée de tension. Il apparait en fait dans les conclusions de la thèse déjà citée, que l'intensité a pour caractéristique de se situer souvent à plusieurs niveaux de l'analyse.

Les phénomènes tels que la focalisation, l'ellipse volontaire, la néologie, l'ironie (antiphrase), l'argot, les gros mots (qu'on qualifie d'ailleurs d'écart de langage, même si gros évoque d'abord la quantité), et autres actes de langage menaçants, l'intonation particulière qui accompagne ces actes que l'intensité caractérise, ressortissent en revanche du contraste virtuel. Chaque fois, on peut mettre en évidence un élément absent avec auquel s'oppose l'élément présent et qui serait plus attendu, non marqué. L'intensité recoupe ici, sans s'y

\footnotetext{
${ }^{9}$ D'après Ducrot, un peu pose l'existence d'une faible quantité, tandis que peu pose la faiblesse d'une quantité existante.

${ }^{10}$ En dehors de cela, comme on sait, tout dans la langue n'est que jeu d'oppositions.

${ }^{11}$ Plus exactement, la deuxième proposition s'oppose à ce qu'implique la première, et qui est implicite.
} 
superposer, ${ }^{12}$ la notion d'information, au sens que lui donne la théorie de l'information. Ainsi, c'est toujours l'idée de mise en opposition, de tension, qui permet de caractériser l'intensité, plus que celle de simple opposition ou d'écart (évoquant simplement ce qui définit la valeur d'un élément au sein d'un système, dans un cadre structuraliste).

Pour résumer mon propos jusqu'à ce point, je propose le schéma suivant. En gras, les termes que je retiens. En italiques, d'autres termes également employés.

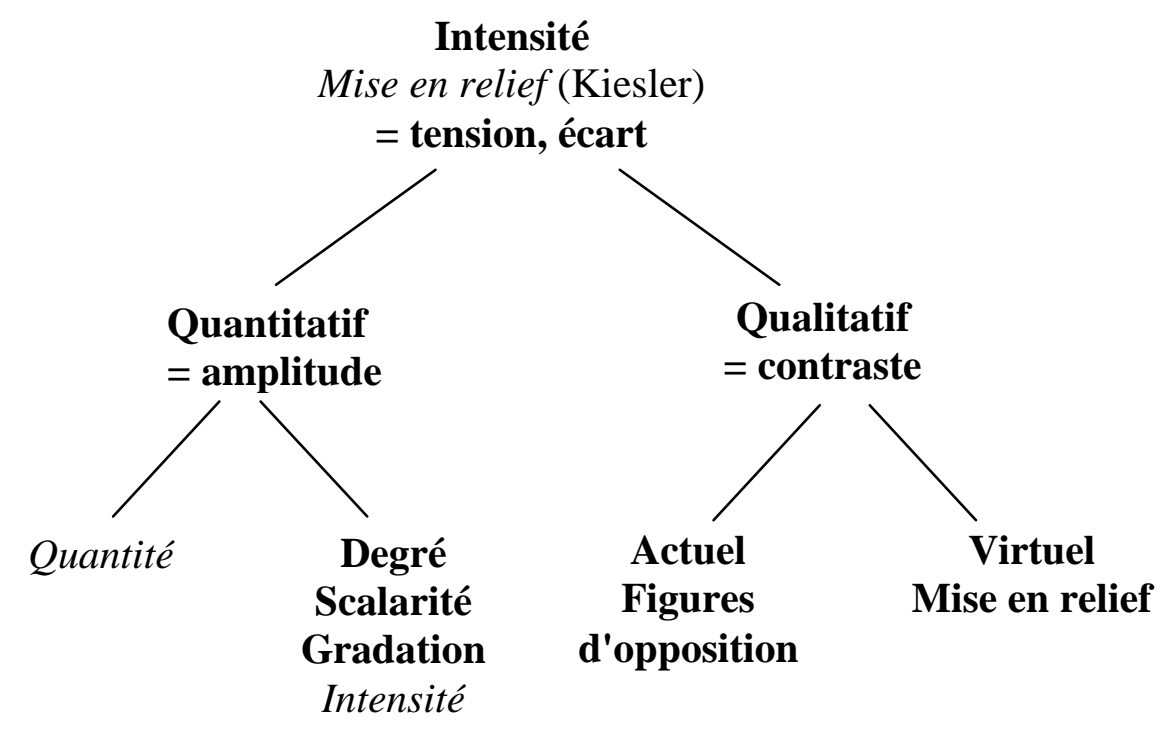

\section{Interférences entre les deux pôles définitionnels de l'intensité}

On a donc fondamentalement deux pôles définitionnels autour desquels se répartissent toutes les modalités d'intensité linguistique : l'intensité de l'ordre de la quantité ou du degré, et l'intensité de l'ordre de la qualité (ou du contraste). Cependant, on a vu au $\S 1$ que des glissements conceptuels étaient possibles. Il n'est donc pas étonnant d'en retrouver sur le plan linguistique. Ainsi, des faits linguistiques relevant de l'un ou l'autre ont-ils pu recevoir la même étiquette (notamment celle de mise en relief ou d'intensité), tandis que certains moyens linguistiques peuvent être employés dans les deux cas. En voici quelques manifestations.

Comme l'a noté Roggero (1979), il existe une expression qualitative linguistiquement grammaticalisée ou figée de la quantité : une belle assiette de charcuterie, un bon kilo, avoir beau, avoir bien mangé, cette dernière étant caractérisée par une double interprétation (quantité-qualité) plutôt que par l'ambigüité. L'adverbe beaucoup est lui même étymologiquement issu d'un tel phénomène, tout comme bien, pas mal, ou même fort.

Bien souvent la mise en opposition est en fait due à un écart quantitatif. Le mot surtout dans Vous m'énervez tous, surtout toi pose tout à la fois une mise en contraste et un écart quantitatif. De même, dans l'énoncé C'est Jean qui va être content!, la focalisation qui a

\footnotetext{
${ }^{12}$ Car c'est le caractère marqué / non marqué, influencé par exemple par des règles de politesse, qui opère plus précisément que la fréquence ou probabilité d'appartition.
} 
habituellement une valeur contrastive (= "C'est moi -et pas le pâtissier-qui ai fait ce gâteau") a en l'occurrence une valeur quantitative : "Jean va être drôlement content".

Dans l'énoncé Il est foutrement borné, l'adverbe intensif en -ment qu'on pourrait comparer à énormément, follement tire, contrairement à ceux-ci, uniquement sa valeur intensive quantitative de son caractère grossier, tabou, contrastant avec la norme de politesse attendue. L'adverbe autrement (dans autrement exigeant), quant à lui, ne fait qu'indiquer le contraste, laissant implicite ce qu'il faut en inférer : une différence qui donne lieu a un écart quantitatif important.

Enfin, l'exclamation, moyen d'expression de l'intensité par excellence, renferme potentiellement les deux aspects de celle-ci : Que c'est beau! (quantitatif, degré, "C'est très beau"), Tu es là toi ! (contrastif, "Je ne m'attends pas à te voir"), Quelle idiote ! (quantitatif), Quelle idée! (qualitatif).

\section{$\underline{\text { 5. L'expression intense est iconique }}$}

Je quitte ici le domaine de la définition de l'intensité pour signaler une de ses caractéristiques intéressantes, qu'est l'iconicité. Dire que l'expression de l'intensité est iconique signifie que quantité et contraste, notions servant à définir sémantiquement l'intensité se retrouvent au niveau de l'expression. Voici quelques lieux où cette iconicité est sensible.

Du côté de l'intensité quantitative, la répétition, l'allongement (dans l'intonation) sont des procédés qui font exclusivement jouer la quantité de signifiant dans le sens d'une augmentation, pour exprimer l'intensité du signifié. D'après Noailly (1999: 56), l'adverbe en -ment, qui peut compter plus de six syllabes est aussi, par une relation iconique entre la quantité de signifiant et l'intensité, à même d'exprimer celle-ci de façon plus marquée encore qu'avec l'adverbe très qui n'en compte qu'une. On constate, en outre, que cette iconicité s'applique à un autre niveau : aux procédés d'intensification eux-mêmes. En effet, il est rare, dans le discours, qu'une marque d'intensité apparaisse isolée. Cette iconicité de l'ordre du quantitatif est illustrée, me semble-t-il, par les expressions métadiscursives : en rajouter, en remettre une couche, qui s'appliquent à celui qui s'exprime intensément, dans la mesure où elles font référence aussi bien singnifié qu'au signifiant.

D'autres phénomènes, phoniques, tiennent à la fois du contraste et de la quantité. La focalisation sur un constituant de l'énoncé s'exprime par une élévation de la hauteur de la voix contrastant avec la hauteur normale, ce qui équivaut à une plus grande fréquence des vibrations : Je l'ai fait, On n'a pas besoin de loi pour ça. De même, lorsque l'on crie pour s'exprimer plus intensément, cela contraste avec le volume normal de la parole et concerne en même temps l'amplitude des vibrations. D'ailleurs, pour représenter graphiquement ce fait, la bande dessinée emploie des caractères gras, lesquels contrastent avec les caractères non gras, en même temps qu'ils requièrent une plus grande quantité d'encre et d'espace. Au contraire, l'ellipse (où du signifiant fait défaut) utilisée comme euphémisme, va dans le sens d'une moindre intensité.

\section{BIBLIOGRAPHIE}


BACRY P., 1992, Les figures de style, Paris, Belin.

CHEVALIER J.-C., BLANCHE-BENVÉNISTE C., ARRIVÉ M. \& PEYTARD J., 1994, Grammaire du français contemporain, Paris, Larousse.

DELELICQ A., 1998, Maths lycée, Paris, Éditions de la Cité.

DUCROT O., 1980, Les échelles argumentatives, Paris, Minuit.

JAKOBSON R., 1963, Essai de linguistique générale, Paris, Minuit.

KIESLER R., 2000, "Où en sont les études sur la mise en relief ?", Le Français moderne, 68-2, p. 224-238.

MOESCHLER J. \& REBOUL A., 1994, Dictionnaire de pragmatique, Paris, Seuil.

NOAILLY, M., 1999, L'adjectif en français, Paris - Gap, Ophrys.

ROGGERO J., 1979, "L'expression qualitative de la quantité", Sigma 4, p. 109-152.

ROMERO C., 2001, L'intensité en français contemporain : analyse sémantique et pragmatique, Thèse de doctorat dirigée par Blanche-Noëlle Grunig, Université de Paris 8. 